\section{Phosphat im Urin}

W. G. Guder

München, Deutschland

Synonym(e) Anorganisches Phosphat im Urin

Englischer Begriff phosphate in urine; phosphaturia

Definition Ausscheidungsrate anorganischer Phosphate im Urin.

Struktur $>$ Phosphat.

Molmasse $>$ Phosphat.

Funktion - Pathophysiologie Anorganische Phosphate werden glomerulär frei filtriert und zu $85-95 \%$ proximal tubulär reabsorbiert. Die Ausscheidungsrate entspricht 5- $15 \%$ der glomerulär filtrierten Menge. Die Phosphatresorption ist abhängig von $>$ Parathormon, $\triangleright$ Calcitonin (hemmen), $\triangleright$ Vitamin D, - Wachstumshormon (fördern). Damit wird die Phosphatausscheidung bei Hyperparathyreoidismus und Calcitoninbildenden Tumoren gesteigert. Phosphate können auch die Grundlage für Harnsteinbildung sein ( $\triangleright$ Struvit), wenn alkalischer Harn-pH vorliegt (z. B. bei Pyelonephritis).

Untersuchungsmaterial - Entnahmebedingungen 24Stunden-Sammelurin, für die Berechnung der tubulären Resorption 2-4 Stunden Urin, angesäuert.
Konventionelle Einheit $\mathrm{mg} / \mathrm{L}, \mathrm{mg} / 24 \mathrm{~h}$.

Internationale Einheit $\mathrm{mmol} / \mathrm{L}, \mathrm{mmol} / 24 \mathrm{~h}$.

Umrechnungsfaktor zw. konv. u. int. Einheit 0,0323 .

Referenzbereich - Erwachsene $13-42 \mathrm{mmol} / 24 \mathrm{~h}$. Fraktionelle tubuläre Resorption $>85 \%(>0,85)$.

Indikation Im Rahmen der $>$ Steinmetaphylaxe, bei Kindern zum Ausschluss einer renal tubulären Resorptionsstörung. Nur noch selten benötigt, da Hormonmessungen aussagekräftiger.

Interpretation Bei Trägern von Struvitsteinen sollte die Phosphatausscheidung $<35 \mathrm{mmol} / 24 \mathrm{~h}$ sein und der $\mathrm{pH}$ $<8,2$ gehalten werden.

Diagnostische Wertigkeit Der diagnostische Wert hat wegen der vielen biologischen Einflussgrößen abgenommen und wurde durch spezifischere Diagnostikmöglichkeiten ersetzt (z. B. PTH-Messung). Auch bei Steinträgern überwiegen die Vermeidung steinbildender Faktoren, insbesondere von Infektionen, sowie die Einhaltung des pH-Werts.

\section{Literatur}

Hesse A, Jahnen A, Klocke K, Nolde A, Scharrel O (1994) Nachsorge bei Harnsteinpatienten. Gustav-Fischer-Verlag, Jena/Stuttgart

Soldin SJ, Rifai N, Hicks JMB (1995) Biochemical basis of pediatric disease, 2. Aufl. AACC Press, Philadelphia

Analytik > Phosphat. 